\title{
ОСНОВНЫЕ ГНОЙНО-ВОСПАЛИТЕЛЬНЫЕ ОСЛОЖНЕНИЯ ПОСЛЕ ВЕНТРОПЛАСТИКИ
}

\section{THE MAIN PURULENT-INFLAMMATORY COMPLICATIONS AFTER VENTROPLASTY}

I. Abuladze

A. Klimov

Summary. Plastic surgery of ventral hernias is one of the most common operations in modern surgical practice. The incidence of purulentinflammatory wound complications during ventral hernia herniation depends on the volume of surgical intervention, the method, material of the endoprosthesis used, the patient's concomitant pathology, and the frequency of the intervention performed. Timely diagnosis of these complications contributes to the implementation of appropriate therapeutic measures aimed at preventing the development of peritonitis and generalization of the infectious process.

Keywords: complications, seroma, fistula, abscess, ventroplasty.

\section{Ввехение}

$\Pi$ роблема лечения вентральных грыж, несмотря на многовековую историю и практику, в настоящее время продолжает оставаться достаточно актуальной. Пластика вентральных грыж является одной из наиболее распространенных операций, составляя $10-21 \%$ от всех оперативных вмешательств в хирургических стационарах [1,2].

Европейское общество по лечению грыж (EHS) подразделяет грыжи брюшной стенки на первичные и послеоперационные. К первой группе относятся средние (эпигастральные и пупочные) и латеральные грыжи, которые классифицируются по размеру $(<2 \mathrm{~cm},>4 \mathrm{~cm}$ и промежуточные). Врожденные или приобретенные пупочные грыжи являются наиболее часто встречающимися среди первичных грыж $[2,3,4]$.

Современные методы вентропластики основаны на имплантации эндопротезов выполняемой во время открытой или, более часто, при лапароскопической хирургии, применение которой способствует достижению более благоприятных послеоперационных результатов (3,7\% от общего числа осложнений) и ограниченной (7,5-10\%) частотой рецидивов заболевания [5].

\author{
Абуладзе Иван Отариевич \\ К.м.н., ГБУ «Городская поликлиника № 22 \\ Департамента Здравоохранения г. Москва» \\ ser.abuladze@yandex.ru \\ Климов Алексей Евгеньевич \\ Д.м.н., профессор, Российский университет дружбы \\ народов
}

Аннотация. Пластика вентральных грыж является одной из наиболее распространенных операций, в современной хирургической практике. Частота возникновения гнойно-воспалительных раневых осложнений при грыжесечении вентральных грыж зависит от объема оперативного вмешательства, способа, материала, используемого эндопротеза, сопутствующей патологии пациента, кратности производимого вмешательства. Своевременная диагностика данных осложнений способствует проведению соответствующих лечебных мероприятий, направленных на предотвращение развития перитонита и генерализации инфекционного процесса.

Ключевые слова: осложнения, серома, свищ, абсцесс, вентропластика.

Раневые инфекции встречаются чаще после выполнения открытого оперативного вмешательства (частота 9-14\%) по сравнению с лапароскопической хирургией (<2\% пациентов) [5,6].

Некоторые авторы полагают, что при использовании сетчатых полимерных эндопротезов (полипропилен, политетрафторэтилен) в лечении вентральных грыж уменьшается количество рецидивов, но увеличивается частота развития раневых осложнений (серома, инфильтрат, краевой некроз кожи, инфаркт подкожно-жировой клетчатки, свищи, гранулемы) [7,8,9,10].

K более серьезным глубоким инфекциям, связанным с выполнением вентропластики и регистрируемым в 2-4\% случаев в популяции относятся: абсцессы и свищи брюшной стенки, кровотечения, непроходимость кишечника, перитонит и рецидивирование грыжи [11].

По мнению большинства авторов, существуют определенные факторы риска, которые способствуют развитию осложнений (общих и местных) в послеоперационном периоде. Среди них: избыточная масса тела (ожирение), наличие сахарного диабета, гипертонии, хронической болезни почек и т.д. Кроме того, такие факторы, как: наличие лигатурных и кишечных свищей, два 

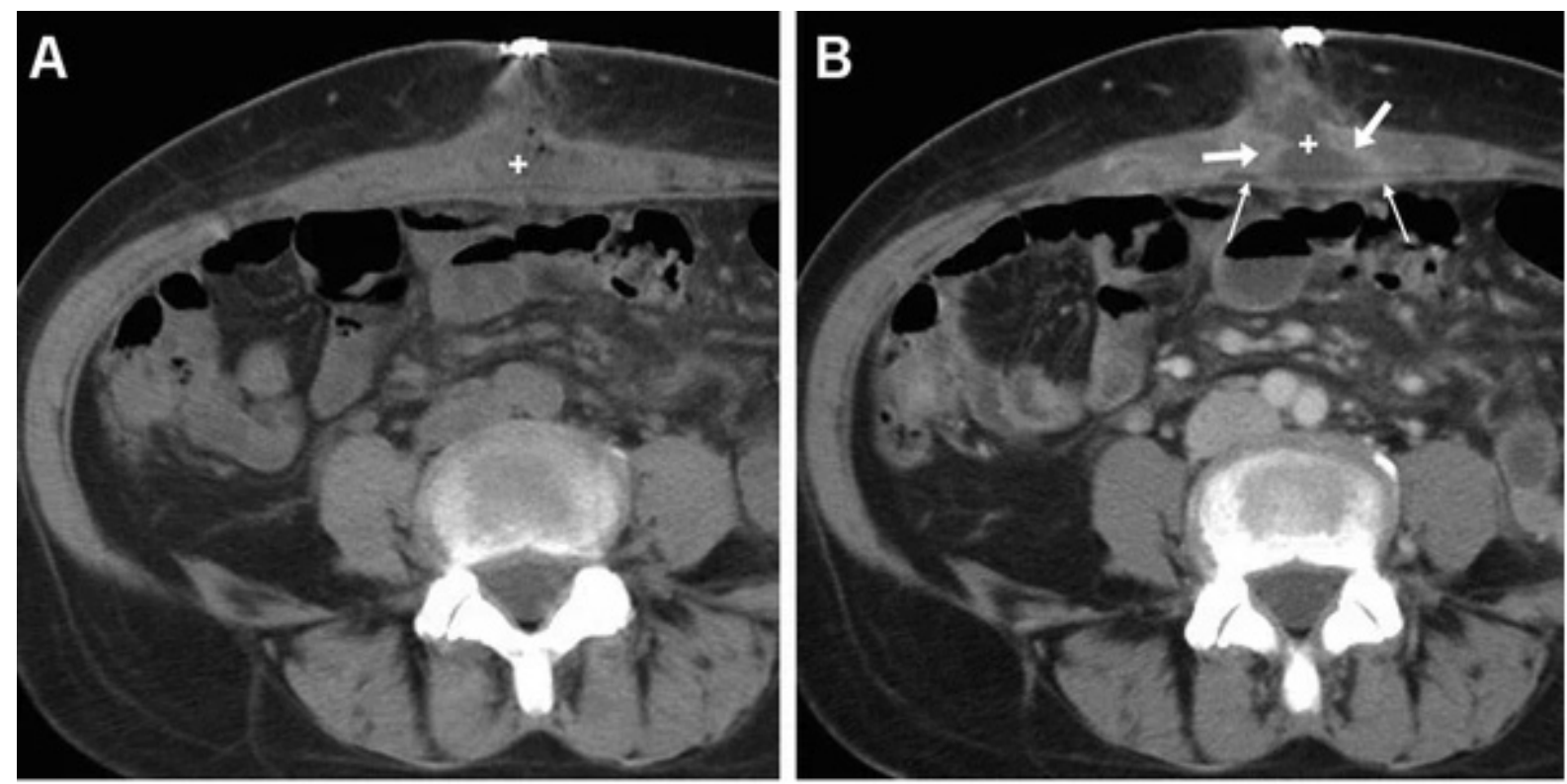

Рис. 1. Вид послеоперационной серомы при проведении компьютерной томографии (КТ) у женщины в возрасте 51 года. Стрелками указано усиление периферического контраста.

грыжесечения в анамнезе, три ранее выполненных операции в брюшной полости, пребывание в больнице 14 дней и более, размер грыжевого дефекта более 300 см2, использование для пластики аутотрансплантата также могут способствовать присоединению инфекции.

Так, например, отмечено, что у пациентов с грыжами и ожирением послеоперационные раневые осложнения возникают в 14-36\% случаев [12]. Анализ литературных данных о развитии возможных гнойно-воспалительных осложнений после выполнения вентропластики будет способствовать разработке современных подходов к лечению и профилактике данной патологии.

\section{Цель работы}

Анализ литературных данных, посвященный вопросам развития возможных гнойно-воспалительных осложнений после выполнения вентропластики.

Осложнения после вентропластики делятся на общие хирургические осложнения и специфические осложнения грыжи. Система классификации Clavien Dindo может быть использована для классификации общих хирургических осложнений, основанных на тяжести и необходимых вмешательствах. Рабочая группа экспертов по вентральной грыже описывает специфические грыжевые осложнения в зависимости от типа хирургического вмешательства (SSO) для стандартизации номенклатуры при изучении результатов после лечения [13].
К наиболее часто встречающемуся осложнению выполнения вентропластики относится развитие серомы. Частота развития данного осложнения определяется способом, объемом и травматичностью производимого оперативного вмешательства. Отмечено, что и при лапароскопическом лечении вентральных грыж, предполагающем минимальную операционную травму и отсутствие отслойки подкожно-жировой клетчатки, развитие сером возможно.

Послеоперационные серомы развиваются на оперированной передней брюшной стенке почти у $10 \%$ пациентов и представляют собой скопление жидкости в тканях передней брюшной стенки вследствие экссудации в потенциальное пространство или полость после оперативного вмешательства. Их образование прежде всего обусловлено развитием неспецифической воспалительной реакции на эндопротез, а также на механическую или химическую травму тканей [14]. Моралес-Конде и соавт. создали систему классификации серомы, в которой серомы были определены как осложнение, только если они имеют симптоматический характер, сохраняются дольше 6 месяцев или становятся инфицированными (типы III и IV). Клинически обнаруженные серомы, которые длятся менее 6 месяцев (типы I и II), классифицируются как случайные данные, отражающие, что они считаются нормальными последствиями операции. Серомы типа III длятся дольше 6 месяцев или становятся симптоматическими, но не требуют вмешательства, в то время как серомы типа IV являются симптоматическими и требуют вмешательства. В этой системе классификации 

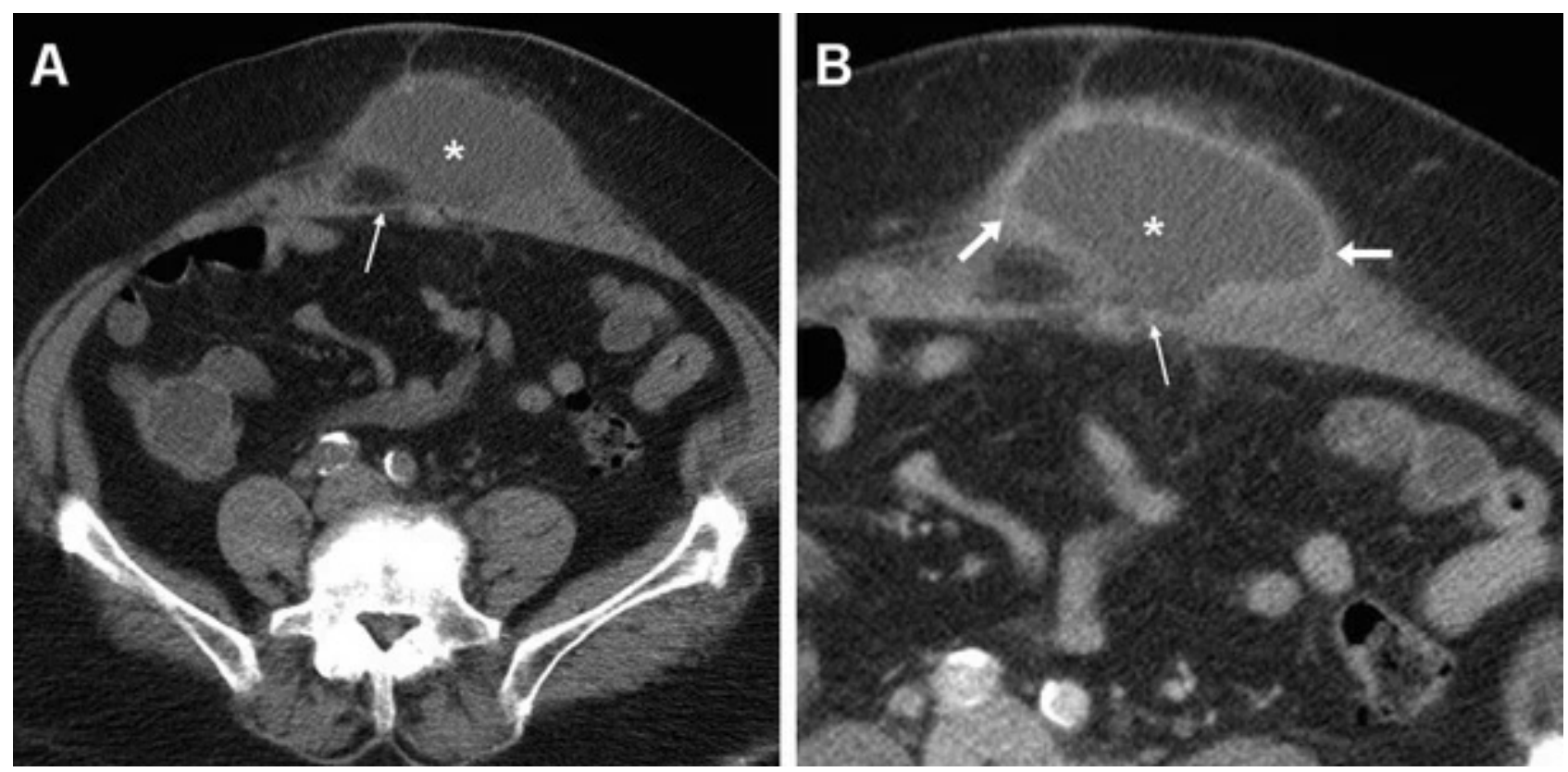

Рис. 2. Схематичное изображение абсцесса при проведении КТ.

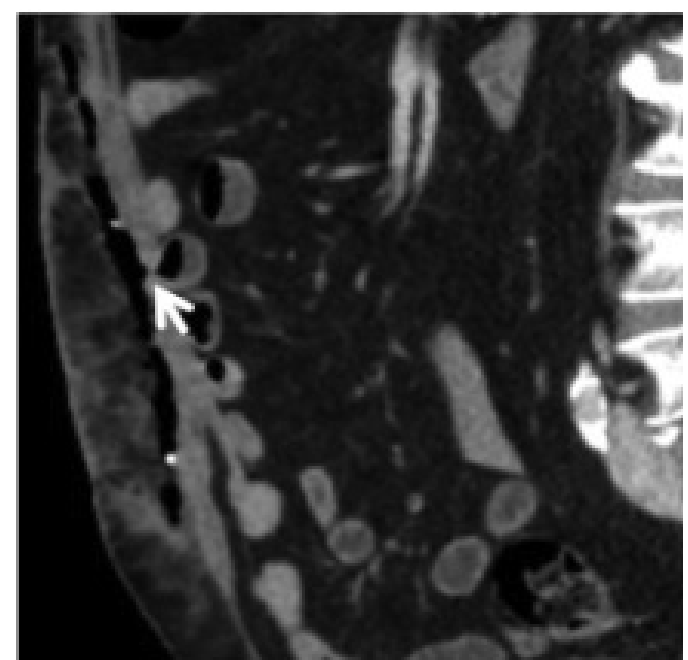

Рис. 3. КТ признаки развития тонкокишечного свища у 60-летней женщины два дня после восстановления стенки с помощью внутрибрюшинной сетки (Parietex $\left.{ }^{\circledast}\right)$. КТ в сагиттальной плоскости подтверждает дефект петли тонкой кишки (короткая стрелка) и наличие подкожного газа, указывающего на развитие свища.

только серомы типа III и IV следует считать истинными осложнениями, поскольку они влияют на клиническое развитие пациента [15].

После выполнения оперативного вмешательства открытым доступом серозная жидкость собирается в ретромускулярно-префасциальном пространстве; и наоборот, после лапароскопической операции серомы возникают в результате протекания перитонеальной жидкости через пористый эндопротез и накапливаются в остаточном грыжевом мешке (рис. 1).

В возникновении серомы важное значение имеет большой грыжевой дефект, остающийся во время операции. Серомы послеоперационной раны после протезирующей герниопластики могут инфицироваться и быть причиной развития нагноений. Некоторыми авторами отмечен факт их возможного увеличения в отдаленные 
сроки после операции, что может симулировать развитие опухоли $[15,16]$.

Абсцесс является наиболее серьезным осложнением. (рис. 2). Абсцессы могут прорваться в свободную брюшную полость с последующим развитием распространенного (диффузного) перитонита. В достаточно редких случаях возможно опорожнение гнойника в полые органы с образованием внутреннего свища. Длительное существование гнойников может привести к абдоминальному сепсису.

Наиболее часто с абсцессом ассоциируется формирование свищей (рис. 3).

Следует отметить, что наихудший прогноз по данным литературы отмечается при высоких свищах в тонком кишечнике, так как это может сопровождаться излитием значительного количества его содержимого с последующим нарушением функций пищеварительной системы [17].

Развитие послеоперационного перитонита, как одного из наиболее опасных и жизнеугрожающих состояний, может быть следствием внутрибрюшных осложнений, а именно: несостоятельности швов анастомозов, периоперационного инфицирования брюшной полости. Данная патология относится к вторичным и третичным формам интраабдоминальной хирургической инфекции [18]. Поэтому особенно актуальны вопросы своевременной диагностики возможных гнойно-воспалительных осложнений при вентропластики, адекватной хирургической тактики и проведения комплексной иммунокоррекции, восстановления физиологических функций органов, обеспечения полисиндромной терапии в послеоперационном периоде.

\section{Bыво $\triangle$}

Внедрение малоинвазивных методов хирургического вмешательства при вентральных грыжах способствует уменьшению развития гнойно-воспалительных осложнений в послеоперационном периоде. Динамическое наблюдение за пациентов, своевременная диагностика раневой инфекции, сером, абсцессов и т.д., комплексный подход в лечении осложнений будут способствовать ранней реабилитации пациентов и достижению положительного клинического результата.

\section{ЛИТЕРАТУРА}

1. Tonolini M, Ippolito S. Multidetector CT of expected findings and early postoperative complications after current techniques for ventral hernia repair // Insights Imaging. 2016 № . 7. P. 541-551.

2. Hindman NM, Kang S, Parikh MS. Common postoperative findings unique to laparoscopic surgery // Radiographics. 2014. № . 34. P. 119-138.

3. Kok ASY, Cheung TSH, Lam DCT, et al. Mesh erosion to urinary bladder causing fistulation to abdominal wall resulting in necrotizing fasciitis: A case report of late complication of incisional hernia // Int J Surg Case Rep. 2017. № . 39. P. 185-187.

4. Picchio M, Muggianu A, Mancini F, et al. Complete mesh migration into the small bowel after incisional hernia repair: a case report and literature review // Acta Chir Belg. 2017. Vol. 117. № . 2. P. 118-121.

5. Endlich M, Schiller W, Mellert F, et al. Implantation of a total abdominal mesh plastic ending up in multiple, lethal right heart injuries // Interact Cardiovasc Thorac Surg. 2015. Vol. 21. № . 1. P. 135-136.

6. Malik AM. Intra-intestinal mesh migration presenting with faecal fistula after incisional hernia repair // J Pak Med Assoc. 2015. Vol. 65.№ . 3. P. 322-323.

7. Лубянский В.Г., Шевченко В. Н., Леонтьев С. В., и др. Влияние различных методов герниопластики на возникновение осложнений // В кн.: Материалы VII Всерос. конф. общих хирургов с международным участием. Красноярск; 2012. с. 336-338.

8. Лембас А.Н., Тампей И. И., Тухарь Г. В., и др. Осложнения протезирующей герниопластики при лечении первичных и послеоперационных вентральных грыж // В кн.: Материалы III междунар. конф. «Современные технологии и возможности реконструктивно-восстановительной и эстетической хирургии». M; 2012; C. 149-151.

9. Паршиков В.В., Чеботарь И. В., Ходак В. А., и др. Парапротезная инфекция В эксперименте in vitro // В кн.: Материалы VII Всерос. конф. общих хирургов с международным участием. Красноярск; 2012; с. 360-363.

10. Молчанов М.А, КривощХков Е. П. Метод пластики грыж передней брюшной стенки с использованием сетчатого импланта // Аспирант. 2015. Т. 4. № . 9. C. 19-21.

11. Мухтаров 3.М., Малков И.С., Алишев О. Т. Профилактика раневых послеоперационных осложнений у больных с послеоперационными вентральными грыжами // Практическая медицина. 2014. Т. 5. № . 81. С. 106-109.

12. Гуменюк, С. Е. Современные методы герниопластики и их осложнения // Национальное здоровье. 2015. № 2. С. 149-156.

13. Dindo D, Demartines N, Clavien PA. Classification of surgical complications: a new proposal with evaluation in a cohort of 6336 patients and results of a survey // Ann Surg. 2004. № . 240. P. 205-213.

14. Köckerling F, Bittner R, Jacob DA, et al. TEP versus TAPP: comparison of the perioperative outcome in 17,587 patients with a primary unilateral inguinal hernia // Surgical Endoscopy. 2015. Vol. 29. № . 12. P. 3750-3760.

15. Morales-Conde S. A new classification for seroma after laparoscopic ventral hernia repair // Hernia. 2012. № . 16. P. 261-267. 
16. Bittner JG. Incarcerated/Strangulated Hernia: Open or Laparoscopic? // Advances in Surgery. 2016. Vol. 50. № . 1. P. 67-78.

17. Хабибрахманова Й.М., Якупова И. Р., Стяжкина С. Н. Кишечные свищи и спаечная болезнь как послеоперационные осложнения // Международный студенческий научный вестник. 2017. № . 2.

18. Керимов Э.Я., Костырной А. В., Керимов Э. Э. Послеоперационный перитонит: практический взгляд на некоторые вопросы // Современные проблемы науки и образования. 2017. № . 6.

с Абуладзе Иван Отариевич, ( ser.abuladze@yandex.ru ), Климов Алексей Евгеньевич.

Журнал «Современная наука: актуальные проблемы теории и практики»

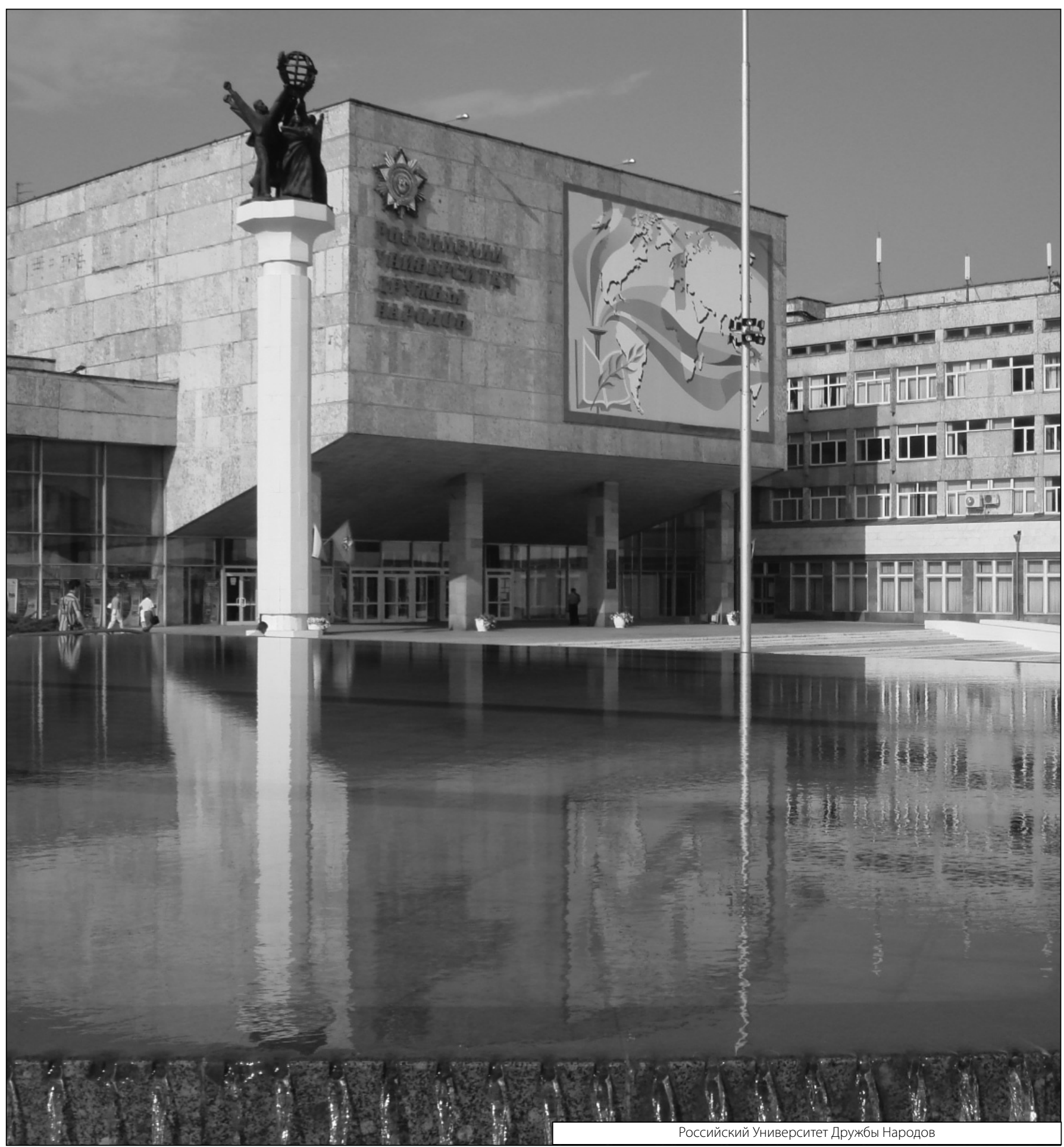

